\title{
The sliding velocity over a sinusoidal bed at high water pressure
}

\author{
Martin Truffer, ${ }^{1}$ Almut Iken ${ }^{2}$ \\ ${ }^{1}$ Geophysical Institute, University of Alaska-Fairbanks, Fairbanks, Alaska 99775-7320, U.S.A. \\ ${ }^{2}$ Rockwinkler Landstrasse 35A, D-28355 Bremen, Germany
}

\begin{abstract}
Under idealized conditions, when pressurized water has access to all low-pressure areas at the glacier bed, a sliding instability exists at a critical pressure, $p_{\mathrm{c}}$, well below the overburden pressure, $p_{0}$. The critical pressure is given by $p_{\mathrm{c}}=p_{0}-\frac{l \tau}{2 \pi a}$, where $l$ is the wavelength and $a$ is the amplitude of a sinusoidal bedrock, and $\tau$ is the basal shear stress. When the subglacial water pressure, $p_{w}$, approaches this critical value, the area of ice-bed contact, $\Delta l$, becomes very small and the pressure on the contact area becomes very large. This pressure is calculated from a force balance and the corresponding rate of compression is obtained using Glen's flow law for ice. On the assumption that compression in the vicinity of the contact arca occurs over a distance of the order of the size of this area, $\Delta l$, a deformational velocity is estimated. The resultant sliding velocity shows the expected instability at the critical water pressure. The dependency on other parameters, such as wavelength $l$ and roughness $a / l$, was found to be the same as for sliding without bed separation.
\end{abstract}

\section{LIST OF SYMBOLS}

$a$

$\mathcal{A}$

$d$

$l$

$\Delta l$

$m$

N

$n$

$p_{\mathrm{c}}$

$p_{0}$

$p_{\mathrm{w}}$

$s$

$s^{*}$

$t_{i j}$
Amplitude of the bedrock undulations

Flow-law parameter

Exponent in sliding law Equation (1))

Wavelength of the bedrock undulations

Ice-bedrock contact area

Exponent in sliding law (Equation (1))

Effective pressure $\left(p_{0}-p_{\mathrm{w}}\right)$

Exponent in Glen's flow law

Critical pressure at which a sliding instability occurs (Equation (2))

Overburden pressure

Water pressure in the cavities

Bed-separation parameter

Dimensionless contact area

Stress-tensor components

Deviatoric stress-tensor components

Basal velocity

Velocity perpendicular to the stoss faces at the contact area

Direction along mean bed

Direction along steepest tangent to the bed

Direction perpendicular upward to the mean bed

Direction perpendicular upward to $x^{\prime}$

Inclination of mean bed

Maximum angle between the mean bed and the stoss faces

Strain-rate components

Basal shear stress

Effective stress

\section{INTRODUCTION}

Sliding over hard bedrock with cavity formation has been a subject of great interest and has been treated extensively (Lliboutry, 1968; Iken, 1981; Kamb, 1987). Lliboutry (1979) and Fowler (1986) have derived sliding laws allowing for bed separation. They have provided graphical solutions for sliding over periodic beds.

Budd and others (1979) and Bindschadler (1983) originally proposed a sliding law of the form

$$
u_{\mathrm{b}} \propto \tau^{m} N^{-d}
$$

to fit observations. Budd's interpretation has been discussed by Lliboutry (1987). Similar laws were found theoretically by Lliboutry (1978) and Fowler (1987). Here, $u_{\mathrm{b}}$ is the sliding velocity, $\tau$ is the basal shear stress, $N$ is the effective pressure (overburden pressure minus water pressure), the exponent $m$ is often set equal to the exponent $n$ in Glen's flow law (in the absence of regelation) and $d$ is an empirical positive number.

This sliding law incoporates an instability as $N$ approaches zero, i.e. the water pressure approaches the iceoverburden pressure.

However, the sliding law in Equation (1) does not allow for an instability at a critical pressure, $p_{\mathrm{c}}$, that is lower than the overburden pressure $p_{0}$. Iken (1981) has shown the existence of such an instability at a pressure

$$
p_{\mathrm{c}}=p_{0}-\frac{\tau}{\tan \beta}
$$

where $\beta$ is the maximum angle between the mean bed and stoss faces. The obvious contradiction between the existence of this critical pressure and the sliding law in Equation (1) was our motivation to derive a different sliding law that incorporates this instability. 


\section{SLIDING VELOCITY NEAR THE GRITICAL PRESSURE}

\section{Assumptions}

To carry out the calculations described below, we need to make the following simplifying assumptions:

(i) We consider a sinusoidal bed of small roughness and large wavelengths (so that regelation is negligible).

(ii) Plane strain is assumed.

(iii) The ice is separated from the bedrock by a thin water film, i.e. the interface supports no shear stresses.

(iv) All cavities contain water at the same pressure.

(v) The water pressure is close to the critical pressure.

We will make the point that assumption (v) means that the contact area between ice and bedrock is very small and is centered around the point of inflection on the stoss faces of the bed undulations. This is not immediately clear, since Gudmundsson (1994) has shown that for a sinusoidal bed (amplitude $a$ and wavelength $l$ ) the pressure maxima are to be found at values of $x$ which solves

$$
\frac{2 \pi}{l} x=\frac{a}{l}+O\left(\left[\frac{a}{l}\right]^{3}\right)
$$

(his equation 4.38). The pressure maxima are at the inflection points for small roughness only. However, Equation (3) was derived for a bed without bed separation. We deal with a situation of extensive bed separation. This affects the stress distribution considerably. In the case of almost complete bed separation, the contact point has to be exactly at the inflection point, because otherwise the instability would occur at a pressure that is even lower than the critical pressure. This can be seen by inspecting the balance of forces on the segment of basal ice shown in Figure la. If the contact area was located at a point different from the inflection point, a new Equation (2) could be derived by a similar analysis. In this new equation, the angle $\beta$ would be replaced by an angle $\beta^{\prime}<\beta, \beta^{\prime}$ being the angle between the mean bed and the tangent at the supposed point of contact. This would lead to an even lower critical pressure. In the same way, one can show that the length of the contact zone must become very small as the water pressure approaches the critical value. Here, we bear in mind that the pressure on a contact area is, by definition, greater than the water pressure.

\section{Stresses at the ice-bedrock contact area}

The analysis is based on a force balance acting on an element of basal ice shown in Figure la. This element extends over one wavelength, $l$, in the $x$ direction, and has a unit thickness in the $y$ direction. The mean stresses along the upper boundary, $\overline{\mathrm{AB}}$, of the element are the macroscopic stresses $p_{0}$ and $\tau$. The lower boundary does not support any shear stress (assumption (iii) above). The normal stress is $p_{\mathrm{w}}$ on the roof of the cavity and there is a mean normal stress of $t_{z^{\prime} z^{\prime}}$ at the contact area. The contact area is defined as that part of the bed where the pressure on the bed is larger than the water pressure in the cavity. It is indicated in Figure lb by a bold line of length $\Delta l$.

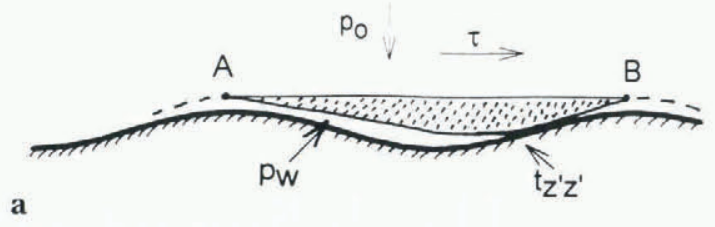

a

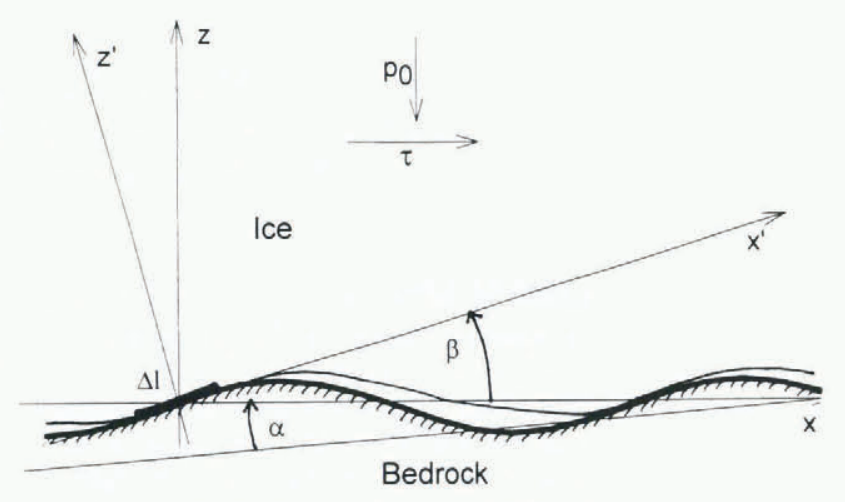

b

1

Fig. 1. Scheme of the glacier base: large, water-filled cavities between ice and sinusoidal bed, which is shown by a hatched line. (a) Element of basal ice (stippled area), to which the force balance refers. The upper boundary of the element, $\overline{\mathrm{AB}}$, is parallel to the mean bed and extends over one wavelength $l$. The lower boundary is the glacier sole between $\mathrm{A}$ and $\mathrm{B} ; \tau$ and $p_{0}$ are the mean stresses on the upper boundary. They are equal to the macroscopic stresses. (b) Coordinate systems. The $x$ axis is chosen along the mean bed of slope $\alpha$, while the $x^{\prime}$ axis is along the steepest tangent to the bedrock. $\beta$ is the angle between the two axes. The area of contact between ice and bed, $\Delta l$, is marked by a bold line. The shapes of the cavities are not exactly known but they are irrelevant for the analysis.

A force balance in the $z^{\prime}$ direction yields the stress component $t_{z^{\prime} z^{\prime}}$, the mean normal stress on the contact area:

$$
t_{z^{\prime} z^{\prime}} s^{*} l=-\tau l \sin \beta-p_{0} l \cos \beta+p_{\mathrm{w}}\left(1-s^{*}\right) l \cos \beta .
$$

$\beta$ is again the largest angle between the mean bed and the actual bed. $s^{*}=\Delta l / l$ is a dimensionless number for the size of the contact area. The first term on the righthand side represents the contribution of the shear stress, the second the contribution of the overburden and the third the contribution of the water pressure to the force balance. The signs in the above equation reflect the fact that the stresses are compressive ( $p_{0}$ and $p_{\mathrm{w}}$ are taken to be positive).

The critical pressure (Equation (2)) is obtained by formulating a similar force balance in the $x^{\prime}$ direction and calculating that water pressure, at which all forces in the $x^{\prime}$ direction balance. This pressure is a limiting value. At higher water pressures, an acceleration along $x^{\prime}$ would result.

The stress calculated in Equation (4) is a principal stress, since the water film does not support any shear stress. The second principal stress is taken to be equal to the water pressure $p_{\mathrm{w}}$. This is correct as long as the contact area is small, as can be seen by calculating the Airy stress function. The limiting case of an infinitely small region has been treated in textbooks on elasticity (e.g. Jaeger, 1971, section 36). 
We can thus calculate the deviatoric stress:

$$
\begin{aligned}
t_{z^{\prime} z^{\prime}}^{\prime} & =\frac{1}{2}\left(t_{z^{\prime} z^{\prime}}-t_{x^{\prime} x^{\prime}}\right) \\
& =-\frac{1}{2 s^{*}}\left(\tau \sin \beta+p_{0} \cos \beta-\left(\left(1-s^{*}\right) \cos \beta+s^{*}\right) p_{\mathrm{w}}\right) .
\end{aligned}
$$

$p_{0}$ can now be replaced by using Equation (2):

$$
t_{z^{\prime} z^{\prime}}^{\prime}=-\frac{1}{2 s^{*}}\left(\frac{\tau}{\sin \beta}+p_{\mathrm{c}} \cos \beta-\left(\left(1-s^{*}\right) \cos \beta+s^{*}\right) p_{\mathrm{w}}\right) \text {. }
$$

\section{Sliding velocity}

Using the above-calculated deviatoric stress and Glen's flow law $\dot{\epsilon}_{i j}=\mathcal{A} \tau_{\text {eff }}^{n-1} t_{i j}^{\prime}$ with $\tau_{\text {eff }}$ given by $\tau_{\text {eff }}^{2}=\frac{1}{2}\left(t_{x^{\prime} x^{\prime}}^{\prime 2}+\right.$ $\left.t_{z^{\prime} z^{\prime}}^{\prime 2}\right)=t_{z^{\prime} z^{\prime}}^{\prime 2}$ we obtain the strain rate $\dot{\epsilon}_{z^{\prime} z^{\prime}}$. This constitutive law is used somewhat arbitrarily since the magnitude and transience of the stress field put it outside the realms of "normal" glacial flow. We now make the additional assumption that the strain rates are effective over a normal distance corresponding to the width of the contact area $\Delta l$. This assumption is justified in a linear theory and approximately applies here. We can therefore use the stresses at the interface to estimate a deformational velocity perpendicular to the bed at the contact area.

$$
\begin{aligned}
u_{\perp} \approx\left|l s^{*} \dot{\epsilon}_{z^{\prime} z^{\prime}}\right|=\frac{\mathcal{A} l \tau^{n}}{2^{n}\left(s^{*}\right)^{n-1}} \\
\left(\frac{1}{\sin \beta}+\frac{p_{\mathrm{c}} \cos \beta}{\tau}-\frac{p_{\mathrm{w}}\left(\left(1-s^{*}\right) \cos \beta+s^{*}\right)}{\tau}\right)^{n} .
\end{aligned}
$$

Note that Equation (7) was derived without using the assumption of a sinusoidal bed.

In steady state, the sliding motion is parallel to the mean bed, so that

$$
u_{\mathrm{b}}=\frac{u_{\perp}}{\sin \beta} .
$$

Using $s^{*} \ll 1$, we get $\left(1-s^{*}\right) \cos \beta+s^{*} \approx \cos \beta$. We can then simplify Equation (7) by using $\frac{p_{c}-p_{w}}{\tau} \cos \beta \ll \frac{1}{\sin \beta}$, which holds at water pressures close to the critical pressure. Equation (7) thus becomes

$$
u_{\mathrm{b}} \approx \frac{\mathcal{A} l \tau^{n}}{2^{n}\left(s^{*}\right)^{n-1}(\sin \beta)^{n+1}} .
$$

This approximate equation is valid for any periodic bed at high water pressures. $\beta$ is the maximum slope of the stoss faces of the bed undulations. These undulations do not have to be sinusoidal. $s^{*}$ is a function of the water pressure with $s^{*} \longrightarrow 0$ as $p_{\mathrm{w}} \longrightarrow p_{\mathrm{c}}$.

In the special case of a sinusoidal bed, the function $s^{*}\left(p_{w}\right)$ can be found. Equation (16) of Schweizer and Iken (1992) provides the required relationship:

$$
p_{\mathrm{w}}=p_{\mathrm{o}}-\frac{l \tau}{\pi a} \frac{\sin (\pi s)+\pi(1-s) \cos (\pi s)}{\sin (\pi s) \cos (\pi s)+\pi(1-s)} .
$$

Their $s$ is the so-called bed-separation parameter and relates to our $s^{*}$ by $s^{*}=1-s$. They obtained their equation in a fashion similar to Lliboutry (1968) but they made a different assumption on the location of the separated zone. They assumed that the separated zone is centered at the inflection point on the lee face of the sinusoidal bump. This assumption applies during the transient phase of the begin- ning of cavity growth and, if the ice is almost fully separated, also for fully developed, steady cavities.

$s^{*}$ can now be calculated by using the assumption of a small contact area $\left(s^{*} \ll 1\right)$. This allows the expansion of sine and cosine in Equation (10) using a Taylor series. Carrying this out yields:

$$
p_{\mathrm{w}} \approx p_{0}-\frac{l \tau}{\pi a}\left[\frac{10-\left(\pi s^{*}\right)^{2}}{20-4\left(\pi s^{*}\right)^{2}}\right] .
$$

For a sinusoidal bed,

$$
\tan \beta=\frac{2 \pi a}{l} .
$$

Thus, as $s^{*} \longrightarrow 0$, the water pressure, $p_{\mathrm{w}}$ approaches the critical pressure, $p_{\mathrm{c}}$, as required.

From Equation (11), we find:

$$
\left(s^{*}\right)^{2} \approx \frac{10\left(p_{\mathrm{c}}-p_{\mathrm{w}}\right)}{\pi^{2}\left(2\left(p_{0}-p_{\mathrm{w}}\right)-\tau \cot \beta\right)} .
$$

Replacing this in Equation (9), we obtain

$$
u_{\mathrm{b}} \approx \frac{\mathcal{A} l \tau^{n}}{2^{n}(\sin \beta)^{n+1}}\left(\frac{\pi^{2}\left(p_{c}+p_{0}-2 p_{\mathrm{w}}\right)}{10\left(p_{\mathrm{c}}-p_{\mathrm{w}}\right)}\right)^{\frac{n-1}{2}} .
$$

Furthermore,

$$
\sin \beta \approx \tan \beta=\frac{2 \pi a}{l} .
$$

This finally gives

$$
\begin{aligned}
u_{\mathrm{b}} & \approx \frac{\mathcal{A} l \tau^{n}}{2^{2 n+1} \pi^{2}}\left(\frac{l}{a}\right)^{n+1}\left(\frac{p_{\mathrm{c}}+p_{0}-2 p_{\mathrm{w}}}{10\left(p_{\mathrm{c}}-p_{\mathrm{w}}\right)}\right)^{\frac{n-1}{2}} \\
& \approx \frac{\mathcal{A} l \tau^{n}}{2^{2 n+1} \pi^{2}}\left(\frac{l}{a}\right)^{n+1}\left(\frac{N}{10\left(p_{\mathrm{c}}-p_{\mathrm{w}}\right)}\right)^{\frac{n-1}{2}} .
\end{aligned}
$$

\section{DISCUSSION}

Equation (16) shows the expected instability as the water pressure approaches the critical pressure. It also preserves the $\tau^{n}$ dependency of the sliding velocity that is typical of any sliding law that describes sliding over hard bedrock in the absence of regelation. A rather appealing fact is that the sliding velocity shows the same dependency on the roughness $a / l$ as in previously derived sliding laws (Kamb, 1970; Fowler, 1979, Gudmundsson, 1994). This was somewhat unexpected, because the above-mentioned authors did a detailed analysis of sliding over bedrock without cavity formation.

Sliding instabilities at pressures below overburden, as predicted here, have not been observed. The simplifying assumption of a sinusoidal bed does not explain this, since the existence of the critical pressure does not depend on that assumption. Also, it is highly unlikely to find many places with stoss faces perpendicular to the mean bed $\left(\beta=90^{\circ}\right)$, which would yield $p_{\mathrm{c}}=p_{0}$. Observations of the glacier bed in front of the present-day terminus of Findelengletscher, for example, show a somewhat smooth bed (Iken and Bindschadler, 1986). At the few places where the stoss faces are perpendicular to the mean bed, a large proportion of the shear stress should be concentrated once the critical pressure is reached and thus accelerated motion should still occur.

We believe that assumption (iv) (all the cavities are at the same water pressure) is the major simplification. Following a recent paper (Iken and Truffer, 1997), we argue that 
there are isolated cavities beneath Findelengletscher that would prevent such an instability.

\section{GONCLUSION}

An approximate sliding law, valid for a sinusoidal bed in the case of extensive bed separation and other idealized conditions, has been derived from a force budget. An important feature of this law, not included in other sliding laws, is the factor $\left(p_{\mathrm{c}}-p_{\mathrm{w}}\right)^{-(n-1) / 2}$. This factor accounts for the instability of sliding that should occur when the subglacial water pressure, $p_{\mathrm{w}}$, approaches the critical value, $p_{\mathrm{c}}$. The dependence of the sliding velocity on bed roughness (amplitude/wavelength), on wavelength and on basal shear stress is the same as for sliding over a sinusoidal bed without bed separation.

\section{ACKNOWLEDGEMENTS}

We thank K. Echelmeyer for reading the manuscript carefully and helping to improve it, and an anonymous reviewer for critical comments.

\section{REFERENCES}

Bindschadler, R. 1983. The importance of pressurized subglacial water in separation and sliding at the glacier bed. f. Glaciol., 29(101), 3-19.
Budd, W.F., P. L. Keage and N. A. Blundy. 1979. Empirical studies of ice sliding. F. Glaciol., $23(89), 157-170$.

Fowler, A. C. 1979. A mathematical approach to the theory of glacier sliding. f. Glaciol., $23(89), 131-141$.

Fowler, A. C. 1986. A sliding law for glaciers of constant viscosity in the presence of subglacial cavitation. Proc. R. Soc. London, Ser. A, 407 (1832), 147-170.

Fowler, A. C. 1987. Sliding with cavity formation. 7. Glaciol., 33 115), 255-267.

Gudmundsson, G. H. 1994. Glacier sliding over sinusoidal bed and the characteristics of creeping flow over bedrock undulations. Eidg. Tech. Hochschule, Zfrich. Versuchsanst. Wasserbau, Hydrol. Glaziol. Mitt. 130.

Iken, A. 1981. The effect of the subglacial water pressure on the sliding velocity of a glacier in an idealized numerical model. 7. Glaciol., 27 (97), 407-421.

Iken, A. and R. A. Bindschadler. 1986. Combined measurements of subglacial water pressure and surface velocity of Findelengletscher, Switzerland: conclusions about drainage system and sliding mechanism. $\vec{j}$. Glaciol., 32(110), 101-119.

Iken, A. and M. Truffer. 1997. The relationship between subglacial water pressure and velocity of Findelengletscher, Switzerland, during its advance and retreat. f. Glaciol., 43 (144), 328-338.

Jaeger, J. C. 1971. Elasticity, fracture and flow: with engineering and geological applications. Third edition. London, Methuen \& Co. Ltd. and Science Paperbacks.

Kamb, B. 1970. Sliding motion of glaciers: theory and observation. Rev. Geophys. Space Phys., 8(4), 673-728.

Kamb, B. 1987. Glacier surge mechanism based on linked cavity configuration of the basal water conduit system. 7. Geophys. Res., 92 (B9), 9083-9100.

Lliboutry, L. 1968. General theory of subglacial cavitation and sliding of temperate glaciers. f. Glaciol., 7 (49), 21-58.

Lliboutry, L. 1979. Local friction laws for glaciers: a critical review and new openings. 7. Glaciol., $23(89), 67-95$.

Lliboutry, L.A. 1987. Very slow flows of solids: basics of modeling in geodynamics and glaciology. Dordrecht, etc., Martinus Nijhoff Publishers.

Schweizer, J. and A. Iken. 1992. The role of bed separation and friction in sliding over an undeformable bed. f. Glaciol., 38(128), 77-92. 